\title{
PERFORMANCE OF A CONNECTIONLESS PROTOCOL OVER ATM
}

\author{
Geert J. Heijenk \\ Aad P. A. van Moorsel \\ Ignas G. Niemegeers \\ University of Twente, Tele-Informatics and Open Systems \\ P.O.Box 217, 7500 AE Enschede, The Netherlands \\ tel. +31 53 893767; fax: +31 53 333815; e-mail: heijenk@cs.utwente.nl
}

\begin{abstract}
Recent studies show the existence of a demand for a connectionless broadband service. In order to cope with this demand, a connectionless protocol for the B-ISDN needs to be designed. Such a protocol should make use of ATM and the ATM Adaptation Layer. It needs to specify destination and bandwidth of connections to the ATM network without advance knowledge of the traffic that has to be transferred over these connections. A possible mechanism which can cope with this problem, the 'On-demand Connection with Delayed Release' (OCDR) mechanism, is described. Its efficient operation is based on the assumption that there exists a certain correlation beiween subsequently arriving $C L$ packets.

Two different arrival processes are used to evaluate the performance of the OCDR mechanism: a Poisson arrival process, and a Markov Modulated Poisson Process (MMPP) which models a bursity traffic source. Markov models of the OCDR mechanism have been constructed for both arrival processes. For the model with Poisson arrivals, a closed form solution is presented. The model with MMPP arrivals is solved numerically.

Compared to a 'Permanent Connection' mechanism significant bandwidth reductions can be obtained provided that the offered traffic has a bursty nature. Furthermore, the $O C D R$ mechanism has the advantageous property that the obtained average node delay is not strongly related to the intensity and burstiness of the offered traffic.
\end{abstract}

\section{Introduction}

The Broadband ISDN (B-ISDN) is being designed in order to satisfy the needs for more bandwidth and more flexibility in public telecommunications in the coming decades. The Asynchronous Transfer Mode (ATM) has been selected as the target transfer mode for B-ISDN. ATM provides a connection oriented $(\mathrm{CO})$ service to its users.

Recent studies however, show the existence of a demand for a connectionless (CL) broadband service ([1], [2]). Applications which might use a connectionless service are for instance LAN and MAN interconnection, electronic mail, telemetry, and Electronic Fund Transfer (EFT). The main feature of a connectionless service is that the service provider and the service user do not make an agreement about the arrival times, the volume, and the destination of traffic. As a result the service provider will not have knowledge about the expected traffic.

The connectionless service will be provided by a connectionless protocol which makes use of the ATM Adaptation Layer (AAL) service. As a result, connectionless service data units (CL-SDUs) have to be transferred over AAL connections. A problem appears in the fact that the protocol has no advance knowledge about the offered traffic, but an agreement has to be made with the AAL in the form of a connection to a particular destination with a certain bandwidth characteristic (e.g. mean and peak bandwidth). These parameters are used by the AAL protocol to establish an ATM connection.

We describe four candidate protocol mechanisms for the transportation of CL-SDUs over AAL connections in Section 2. In order to be able to evaluate the performance of one of the mechanisms, Markov models have been constructed. This mechanism has been evaluated for different arrival processes, a Poisson process and a Markov Modulated Poisson Process (MMPP). These are discussed in the section on workload (Section 3). The constructed models, one for Poisson arrivals and one for MMPP arrivals, are described in Section 4. The results obtained from the analysis of the models are discussed in Section 5. Finally, in Section 6 conclusions are drawn.

\section{Protocol}

Basically, two different protocols can be used to provide a connectionless service over ATM: depending on the application either an end-to-end protocol or a node-by-node protocol can be used ${ }^{1}$. The end-to-end protocol (see Figure 1) corresponds to the case with connectionless protocol entities only in the end systems. In order to transfer a CL-SDU from one end system to another end system, use should be made of an AAL connection between these end systems. The node-bynode protocol (see Figure 2) corresponds to the presence of one or more intermediate CL protocol entities. If an end system has to transfer a CL-SDU, it has to use an AAL connection to an intermediate node. In this intermediate node, the data unit is routed to a connection with the appropriate next node, and so on. Finally, in the destination node, the CL-SDU is delivered to the receiving service user.

\footnotetext{
I In CCITT draft recommendation I.211 ([3]) these two ways of providing a connectionless service are called indirect (end-to-end) and direct (nodeby-node).
} 


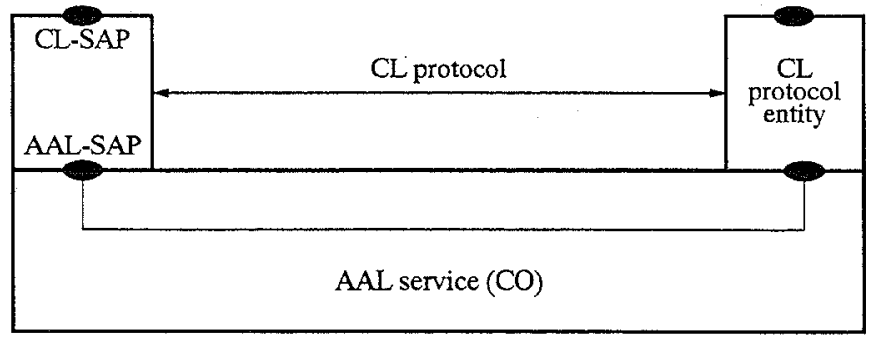

$C L=$ Connectionless

$\mathrm{CO}=$ Connection Oriented

$\mathrm{AAL}=\mathrm{ATM}$ Adaptation Layer

SAP $=$ Service Access Point

Figure 1: Connectionless service with an end-to-end protocol

As stated before, AAL connections are used to transfer CL-SDUs. Before a $\mathrm{CL}$ protocol entity can use an $\mathrm{AAL}$ connection, it has to establish the connection and specify the relevant destination protocol entity and the required bandwidth. Here arises a problem: the CL protocol entity does not have advance knowledge about the destination and the required bandwidth since the provided service is connectionless. However, it has to specify those to the AAL layer in order to establish a connection. To solve this problem we make use of assumed correlation between subsequently arriving CL-SDUs.

A certain correlation, in the destination as well as in the arrival time, can be expected due to the behaviour of (application) protocols in the higher layers. This expected correlation can be used to specify the destination or bandwidth of an AAL connection. For example, the expectation that there is a large amount of traffic between two LANs which have to be interconnected, can lead to the establishment of a more or less permanent connection between these LANs.

Several mechanisms for setting up AAL connections for the transfer of CL-SDUs can be identified (From now on we will use packet for CL-SDU):

\section{Connection per Packet}

A connection, necessary to transfer a packet, can be established as soon as the packet arrives at a protocol entity. The connection is released again immediately after the transfer of the packet. This mechanism does not use expected correlation between packets. All necessary knowledge, i.e. destination and amount of data to be transported, is known

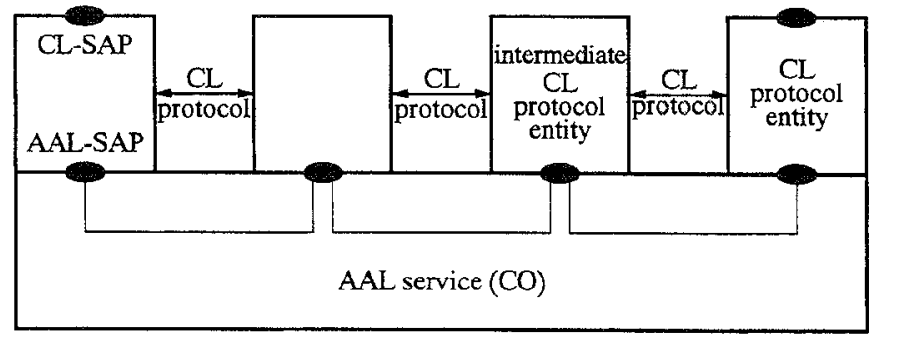

$\mathrm{CL}=$ Connectionless

$\mathrm{CO}=$ Connection Oriented

$\mathrm{AAL}=\mathrm{ATM}$ Adaptation Layer

SAP $=$ Service Access Point

Figure 2: Connectionless service with a node-by-node protocol at the moment the connection is established. Note that a packet will in general be sent in several ATM-SDUs (cells). A practical extension is to transfer subsequent packets, arriving during the transmission time of the current packet, over the same connection.

\section{Permanent Connection}

A CL protocol entity could have one or more permanent connections to possible destinations for $\mathrm{CL}$ traffic, and transfer a packet on one of these connections. The specification of the required bandwidth is a problem for this solution. In principle, the CL protocol entity has no knowledge about the arrival times and the lengths of the packets.

3. On-demand Connection with Delayed Release (OCDR)

According to this mechanism an AAL connection will be established if a packet is offered, and no connection is available. The connection will not be released immediately after sending the packet. It can be used for consecutive packets as well. The connection will be released if it is not used for a certain period, the holding time. The aforementioned mechanisms are special cases of this one. The OCDR mechanism with holding time zero is equivalent to the 'Connection per Packet' mechanism with the mentioned extension. The 'Permanent Connection' mechanism is equivalent to the OCDR mechanism with an infinite holding time.

\section{Variable Bandwidth Connection}

In ATM it will be possible to change the bandwidth of a connection during the lifetime of a connection. This mechanism makes use of that property. Initially, no connection will be available. As soon as a packet arrives, a connection to the relevant destination will be established. The bandwidth of this connection will be relatively low. Consecutive packets with identical destination will be transferred over the same connection. If necessary, for instance when several packets are waiting to be served in a buffer, the CL protocol entity will request the ATM network to increase the bandwidth. If no longer necessary the entity will, after a certain holding time, request the ATM network to decrease the bandwidth, or release the connection. Both actions, increasing bandwidth and decreasing bandwidth, can be repeated several times.

The choice for a certain mechanism and the accompanying parameters (e.g. holding time, bandwidth levels) can be made for each protocol entity individually. The choice will depend on the expected arrival and transmission time distribution of packets (i.e. the expected applications). Furthermore, it will depend on the possibility to multiplex connectionless traffic from several sources onto one outgoing connection. In general, this will be the case for intermediate nodes, where connectionless traffic from different incoming connections can have the same destination.

In the sequel, we will model and analyse the OCDR mechanism. The modelling and analysis of the 'Variable Bandwidth Connection' mechanism can be found in [4]. 


\section{Workload}

For the performance analysis of a CL protocol, we need to define the workload on the protocol. Here, we characterize all traffic, which has to be transferred from a certain source node to the same destination node, as a stochastic process. These nodes can be either end or intermediate nodes.

We adopt two different workloads in this paper. The first one, Poisson traffic, is a very simple one. We will later see that it results in an analytically tractable performance model. It gives insight in the behaviour of the protocol. The second one, bursty traffic, is a more complex one. It gives a more realistic characterization of the expected traffic, and therefore more accurate performance measures.

\subsection{Poisson Traffic}

The first workload description, used for our performance analysis, is a Poisson arrival process. The interarrival time of packets is exponentially distributed with mean $1 / \lambda$. We are able to derive a closed form solution for our model using this workload. Therefore, modelling with this workload gives insight in the behaviour of the CL protocol.

The well operation. of the OCDR mechanism is based on the assumption that there will be a correlation in arrival times of subsequently transferred packets. Poisson traffic does not have this property. Therefore, the advantageous qualities of the mechanism will not be revealed maximally. The analysis of the mechanism with. Poisson traffic can therefore be considered as a worst case analysis.

\subsection{Bursty Traffic}

In order to have a workload, which exhibits the assumed correlation between subsequently arriving packets, we define a second stochastic process. It assumes that packets arrive in bursts (see Figure 3 ). The traffic is characterized by a burst time, an interburst time, and an interarrival time. In [5], it is shown that such a bursty traffic source, which is modelled by a "Train Model", provides a realistic description of the traffic on a local computer network (Ethernet). In [6], it is shown that a Markov Modulated Poisson Process (MMPP), which can also be used to describe the bursty traffic source is very well suited to represent correlations between subsequent arrivals. We prefer to model the traffic source with an MMPP. This arrival process approximates the "Train Model", and has the advantage of allowing for Markovian analysis. We assume the burst time, the interburst time, and the interarrival time to be exponentially distributed, with means $1 / \alpha, 1 / \beta$, and $1 / \lambda$ respectively. Our MMPP has two states: burst and interburst. It transits with rate $\alpha$ from state burst to state interburst, and with rate $\beta$ in the opposite direction. If the MMPP is in state

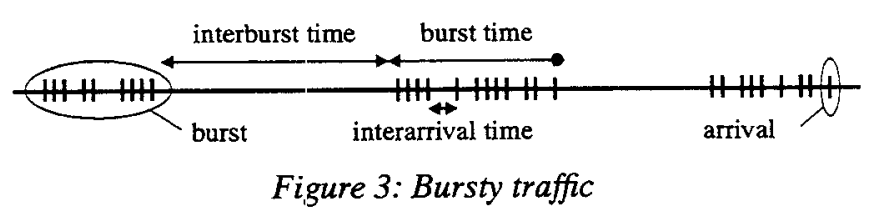

burst, packets arrive with rate $\lambda$. In state interburst, no arrivals take place.

\subsection{Packet Length Distribution}

For both arrival processes, we adopt the same packet length distribution. We assume the packet length to be exponentially distributed. The mean packet length is $l$ bits.

\section{Markov Model of the OCDR mechanism}

In this section we discuss the performance modelling of the above described OCDR protocol. Metrics of interest for this protocol are for instance the reserved bandwidth, the number of connection setups per second, mean delay and loss probability. First, we present a Markov model for the OCDR mechanism with Poisson arrivals. We derive a closed form solution for the stationary state probabilities of this model. Next we present a Markov model for the OCDR mechanism with bursty arrivals. Table 1 gives an overview of the parameters used in the models.

\subsection{Poisson Traffic}

The description of our Markov model is as follows. We focus on one station and assume that packets arrive at the station according to a Poisson process with rate $\lambda$. When a packet arrives the connection setup procedure is started. Setting up a connection takes an exponentially distributed time with mean $1 / \mathrm{c}$. When the connection has been established the transmission of packets can be started. We assume that for every packet transmission takes an exponentially distributed time with mean $1 / \mu$. When all packets have been sent, the connection can be released. The connection will however only be released when the system is empty for an exponentially distributed holding time with mean $1 / r$. In Figure 4 the resulting state transition diagram is depicted.

With state $(i, j)$ we indicate that the number of packets waiting for transmission over the established AAL connection equals $i$, while $j=0$ or $j=1$ indicates that a connection is not or is established respectively.

To compute metrics like mean delay or mean reserved bandwidth we need the steady-state probability distribution of the described Markov chain. For this model the following system of balance equations holds:

$$
\begin{gathered}
(c+\lambda) P(i, 0)=\lambda P(i-1,0) \text { for } i \geq 1, \\
\mu P(i, 1)=\lambda(P(i-1,1)+P(i-1,0)) \text { for } i \geq 1, \\
r P(0,1)=\lambda P(0,0),
\end{gathered}
$$

and, for normalization:

$$
\sum_{i=0}^{\infty}(P(i, 0)+P(i, 1))=1 .
$$

We will relate all stationary probabilities to $P(0,0)$, and use the following notation: 


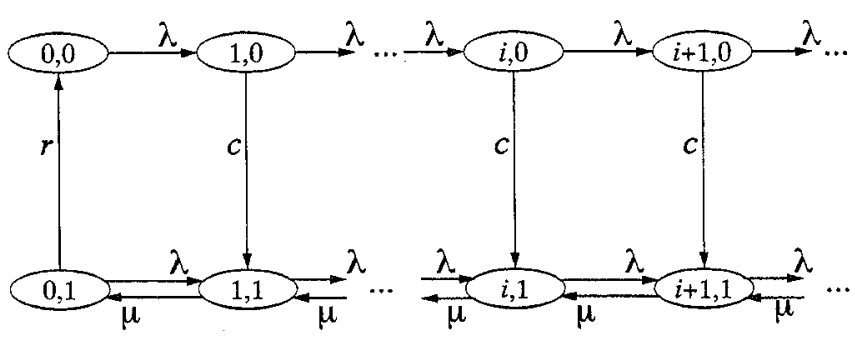

Figure 4: Markov model for OCDR with Poisson arrivals

$$
\rho=\frac{\lambda}{\mu} \text { and } \sigma=\frac{\lambda}{\lambda+c} .
$$

From (1) it follows directly that, for all $i$ :

$$
P(i, 0)=\sigma^{i} P(0,0) \text {. }
$$

From (2) we have for the states $(i, j)$ with $j=1$ :

$$
\begin{aligned}
P(i, 1) & =\rho\left(P(i-1,1)+\sigma^{i-1} P(0,0)\right) \Rightarrow \\
P(i, 1) & =\rho^{i} P(0,1)+\sum_{k=1}^{i} \rho^{k} \sigma^{i-k} P(0,0) .
\end{aligned}
$$

Using (3) this results, for all $i$, in:

$$
P(i, 1)=\left(\frac{\lambda}{r} \rho^{i}+\sum_{k=1}^{i} \rho^{k} \sigma^{i-k}\right) P(0,0) .
$$

With the help of the Cauchy product rule we now could obtain a closed form expression for the steady state probability distribution of the Markov chain, by solving the normalization equation (4) for $P(0,0)$. However, a more elegant solution is obtained when we realise that the fraction of time a server is busy equals the utilization $\rho$, i.e. the following equality holds:

$$
\sum_{i=1}^{\infty} P(i, 1)=\rho \text {. }
$$

Then it follows with (3), (5) and (6), from the normalisation equation (4):

$$
P(0,0)\left(\sum_{i=0}^{\infty} \sigma^{i}+\frac{\lambda}{r}\right)+\rho=1
$$

\begin{tabular}{|c|l|}
\hline $1 / \alpha$ & average burst time \\
$1 / \beta$ & average interburst time \\
$1 / \lambda$ & average packet interarrival time \\
$1 / \mu$ & average packet transmission time \\
$1 / r$ & average connection holding time \\
$1 / c$ & average connection setup time \\
$l$ & average packet length \\
\hline
\end{tabular}

Table 1: Notation

$$
\Rightarrow \frac{1}{(1-\rho)}\left(\frac{1}{1-\sigma}+\frac{\lambda}{r}\right) P(0,0)=1,
$$

which after rewriting results in the following expression for $P(0,0)$ :

$$
P(0,0)=(1-\rho) \frac{1 / \lambda}{1 / \lambda+1 / r+1 / c} .
$$

Substitution of this expression in (5) and (6) leads to a closed form solution for the stationary state probabilities.

Having deduced this closed form solution we can compute the following metrics which we will later use in the evaluation of the OCDR mechanism.

- Average node delay, ET, using Little's law and the Cauchy product:

$$
\begin{gathered}
E T=\frac{1}{\lambda} \sum_{i=0}^{\infty} i(P(i, 0)+P(i, 1))= \\
=\frac{1}{\lambda}\left(\frac{\rho}{1-\rho}+\left(\frac{\lambda+c}{c}\right) \frac{1 / c}{1 / \lambda+1 / r+1 / c}\right) ;
\end{gathered}
$$

- Average reserved bandwidth, $E B W$, using (7):

$$
\begin{gathered}
E B W=l \mu \sum_{i=0}^{\infty} P(i, 1)= \\
=l \mu\left(\rho+(1-\rho) \frac{1 / r}{1 / \lambda+1 / r+1 / c}\right) ;
\end{gathered}
$$

- Number of connection setups per second, EC:

$$
\begin{aligned}
& E C=c \sum_{i=1}^{\infty} P(i, 0)= \\
& =(1-\rho) \frac{1}{1 / \lambda+1 / r+1 / c} .
\end{aligned}
$$

\subsection{Bursty Traffic}

The Markov model for the OCDR mechanism with bursty arrival process (MMPP) is depicted in Figure 5. The state space now is a three tuple $(i, j, k)$. As before, $i$ denotes the number of jobs in the buffer and $j$ denotes whether the connection is up $(j=1)$ or down $(j=0)$. The parameter $k$ represents whether the arrival process is in a burst $(k=1)$ or not $(k=0)$. When the process is in burst mode, it generates traffic according to a Poisson process with rate $\lambda$, otherwise it generates no traffic. The parameter $\mu$ again denotes the packet transmission rate and the parameters $\alpha$ and $\beta$ denote the rates of the exponential burst and interburst time distribution respectively.

We see from Figure 5 that given that the process is in burst, the model is identical to the one for Poisson traffic (Figure 4). Unfortunately, the modulation of the Poisson process complicates the model to such extent that we were not able to find a closed form solution as we did for the model with Poisson 


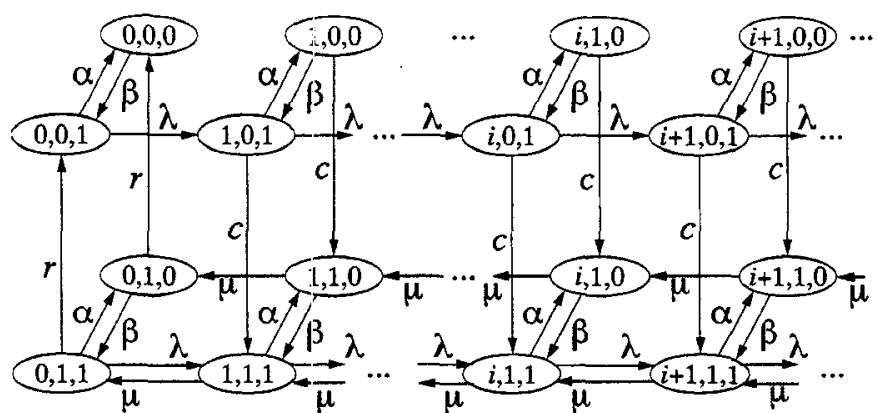

Figure 5: Markov model for OCDR with MMPP arrivals

traffic. Therefore we have solved the balance equations numerically with Gaviss Seidel iteration. To apply this numerical method we have to assume a bounded number of states. We will take a bound for which the resulting buffer full probability is small. In the results presented in this paper we have taken a bound of 100 packets in the queue, resulting in a buffer full probability of lesis than $10^{-5}$. Having obtained the steady state distribution we can derive performance measures by summation of the steady-state probabilities over the appropriate sets of states, as in Section 4.1.

\section{Evaluation}

Using the models described in Section 4, we will evaluate the performance of the OCDR mechanism. This will be done by comparing it to the 'Connection per Packet' and 'Permanent Connection' mechanisms (see Section 2). The following measures are of interest for the evaluation of the OCDR mechanism:

- average node delay, ET

The node delay is the time elapsing from the arrival of (the first bit of) a packet in the CL protocol entity until the departure of (the last bit of) the packet. This delay consists of the time a packet spends in a buffer plus the time necessary for transmission of a packet on an outgoing connection.

- average reserved bandwidth, $E B W$

The reserved bandwidth will be higher than the actually used bandwidth. It is defined as the fraction of time, a connection is present, times the bandwidth of that connection $(\mu l)$. The average reserved bandwidth will be strongly related to the costs of the service.

- average number of connection setups per second, $E C$

The establishment of a connection requires signalling, i.e. the exchange of messages between nodes and processing in the nodes. Therefore the average number of connection setups per second is also a measure related to costs.

In the following, we will first give the values for the workload parameters that have been used. Then we will evaluate the performance of OCDR with a workload of Poisson traffic as well as of bursty (MMPP) traffic.

\subsection{Workload Parameters}

The workload parameters for bursty traffic are based on measurements in [5], taking into account the high-speed character of future applications. The average interburst time has been taken 25 seconds, i.e. $\beta=0.04$. The average burst time is 1 second, i.e. $\alpha=1$. The average number of arrivals per burst is 100 , i.e. $\lambda=100$. This is an order of magnitude higher than the value measured in [5], because of the expected increase in traffic intensity in the future.

For Poisson traffic, we only have to define the parameter of the exponential distribution of the interarrival time, $\lambda$. In order to have an average number of arrivals per second equivalent to bursty traffic, we have adopted

$$
\lambda=100 \frac{\alpha}{\alpha+\beta}=\frac{100}{26} .
$$

The average packet length, $l$, is assumed to be $10 \mathrm{kbits}$ for both Poisson and bursty traffic. The average of the connection setup time, $1 / c$, is assumed to be $100 \mathrm{~ms}$.

\subsection{Poisson Traffic}

The expected gain of the OCDR mechanism is that it needs to reserve less bandwidth than a 'Permanent Connection' mechanism to obtain a certain average node delay. Therefore, an interesting curve is the average node delay versus average reserved bandwidth curve (see Figure 6). Curves are depicted for different holding times $1 / r$ with $r=10000, r=10$, and $r=1$, and for a 'Permanent Connection' mechanism $(r=0)$. For each individual curve, the only varying parameter is $\mu$, and we thus have plotted the results from (9) versus (11) for a range of $\mu$. We therefore introduce the notation $E T(\mu, r)$ and $E B W(\mu, r)$ to show the dependence of $E T$ and $E B W$ on the parameters $\mu$ and $r$. As an example, the figure provides us the following information. Say that we want to have a maximum delay, e.g. $E T(\mu, r)=0.05$, then we can look in the figure which holding time needs the least bandwidth reservation. In this case the permanent connection $(r=0)$ is the best choice.

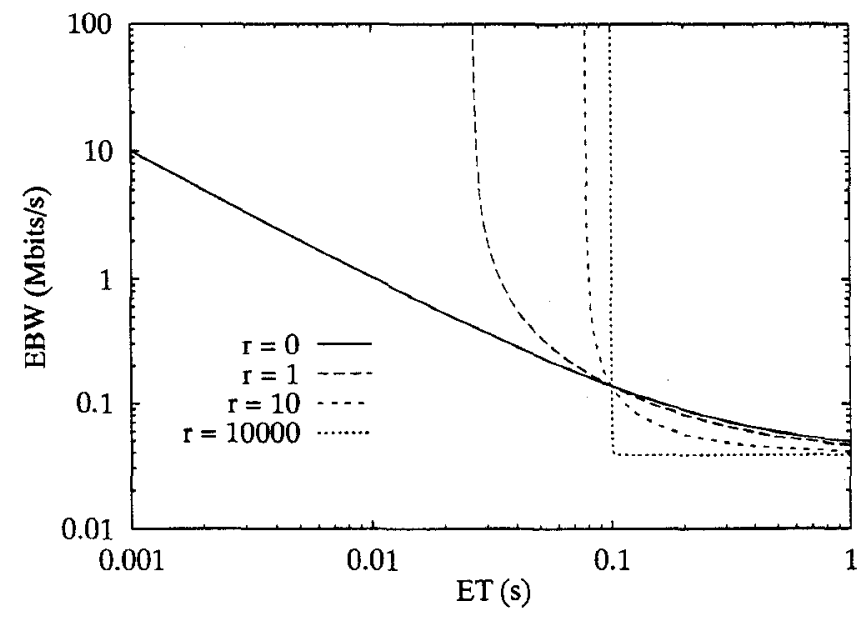

Figure 6: Required bandwidth to obtain a certain average node delay (Poisson traffic) 
We will now discuss the characteristics of the graph in more detail. All the curves show an asymptotic behaviour to some limit value of $E T$. This is the limiting behaviour for $\mu \rightarrow \infty$, and it can be shown that the asymptotic value is as follows:

$$
\lim _{\mu \rightarrow \infty} E T(\mu, r)=\frac{1}{c} \frac{(1 / \lambda+1 / c)}{(1 / \lambda+1 / r+1 / c)} .
$$

We directly see that this implies for the 'Connection per Packet' mechanism $(r=\infty)$ that the limiting value equals $1 / c$, the setup time. For the 'Permanent Connection' mechanism every delay demand can be guaranteed, as the delay goes to 0 when the transmission rate $\mu$ goes to infinity. Of course, the practical bound here is the peak transmission rate.

We see from the curves that the optimal value for $r$ for some mean delay is either $r=0$ when $E T(\mu, r)<1 / c$, or $r \rightarrow \infty$ when $E T(\mu, r)>1 / c$. The average reserved bandwidth then is respectively:

$$
E B W(\mu, 0)=l \mu \text { and } E B W(\mu, \infty)=l \lambda .
$$

Furthermore it can be shown that in the point $E T(\mu, r)=1 / c$ the reserved bandwidth is independent of the holding time and equals $E B W(\mu, r)=l(\lambda+c)$. So, we see that the optimal bandwidth reduction from the OCDR mechanism is about $l c$, the difference between the bandwidth for the 'Permanent Connection' and the 'Connection per Packet' mechanism near $E T=1 / c$. This is a reduction of $72 \%$. It is important to note the difference between reserved bandwidth, used bandwidth and transmission rate. The reserved bandwidth is the transmission rate multiplied with the time it is available. The used bandwidth equals $l \lambda$ and is a lower bound for the reserved bandwidth. In the point $E T(\mu, r)=1 / c, E B W(\mu, r)=l(\lambda+c)$, where all the lines cross, the transmission rate that is used to have this delay and reserved bandwidth is dependent of $r$ :

$$
\mu=(\lambda+c)(1+r / \lambda) .
$$

Concluding we can state that the OCDR mechanism is not advantageous for the model with a Poisson arrival process, since either the 'Connection per Packet' or "Permanent Connection' mechanism needs the least bandwidth to fulfil some delay demand. This is due to the fact that the Poisson process does not exhibit any correlation between subsequent arrivals. In the following section we will evaluate the OCDR mechanism for a Markov Modulated Poisson Process in which this correlation does exist.

\subsection{Bursty Traffic}

We start analysing the OCDR mechanism under bursty traffic with the same type of graph as given for Poisson traffic: the average node delay is plotted versus the average reserved bandwidth (see Figure 7). Curves have been drawn for $r=100, r=10, r=1, r=0.1$, and $r=0$ (permanent connection). Again, the varying parameter is $\mu$.

Contrary to the OCDR mechanism under Poisson traffic, the mechanism under bursty traffic is advantageous.

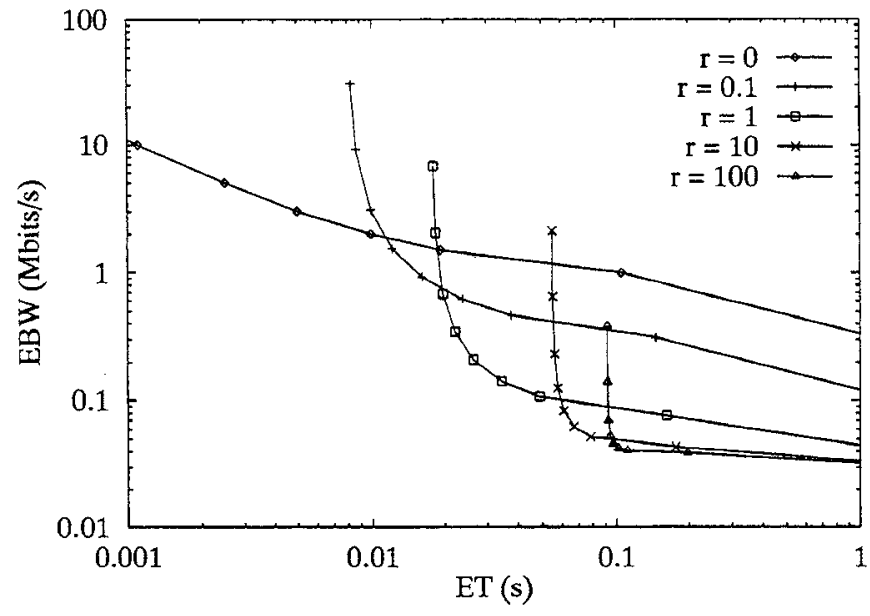

Figure 7: Required bandwidth to obtain a certain average node delay (bursty traffic)

Depending on the required average node delay, one of the values for $r$ yields the lowest average reserved bandwidth. For the given parameters, it can roughly be said that $r=0$ is the optimal solution for $E T<0.01$, and $r \rightarrow \infty$ for $E T>0.1$. For $0.01<E T<0.1$, intermediate values for $r$ are optimal. For this particular workload parameters, bandwidth reductions up to $96 \%(E T=0.1, r \rightarrow \infty)$ can be obtained.

An average node delay in the range from 10 to $100 \mathrm{~ms}$ will probably be the operational region for a connectionless protocol over ATM, since future requirements can be expected to be in this order of magnitude. This makes the bandwidth reduction property of OCDR in this range a realistic advantage.

In order to examine the behaviour of the OCDR mechanism under varying load and burstiness, we give some graphs where the system parameters $r$ and $\mu$ are such that we obtain an average node delay of $25 \mathrm{~ms}$. We do this both for $r=0$ (permanent connection) and for $r=1$ (most optimal curve for $E T=0.025$ ). The corresponding values for $\mu$ are 138 and 336 respectively. Note that this means that the OCDR mechanism needs a higher peak bitrate for these parameters.

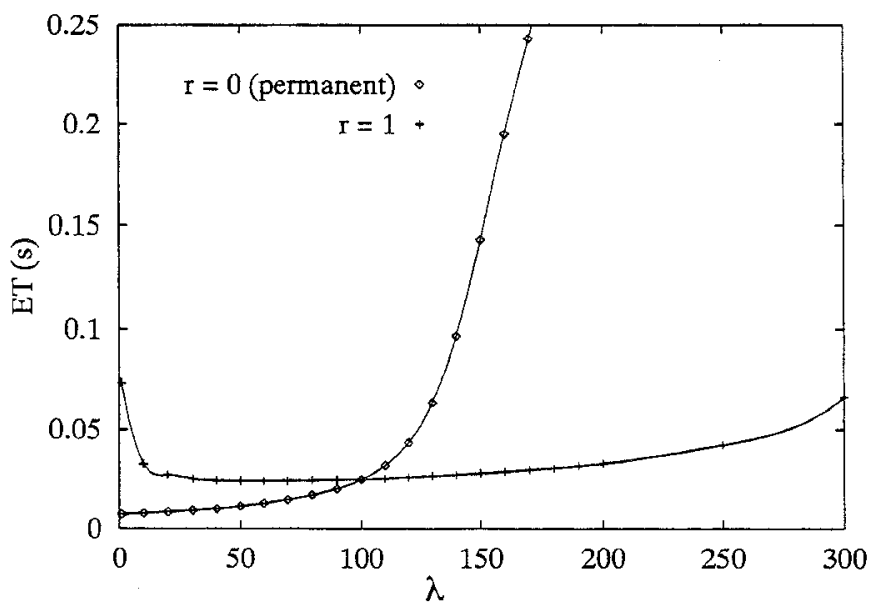

Figure 8: Average node delay under varying load 


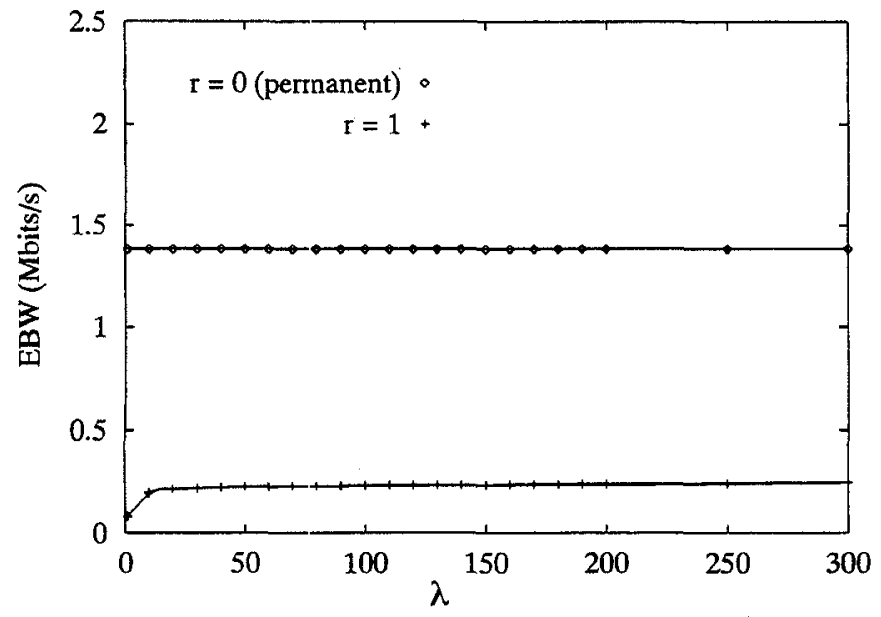

Figure 9: Average reserved bandwidth under varying load

\section{Behaviour under varying load}

In order to vary the load on the connectionless protocol, $\lambda$ is varied. Increasing $\lambda$ means that the interarrival time in a burst decreases, and the number of arrivals per burst increases. Graphs are given for the average node delay ET (Figure 8), the average reserved bandwidth $E B W$ (Figure 9), and the average number of connection setups per second $E C$ (Figure 10).

From Figure 8 , it can be observed that sensitivity of the average node delay to the offered load is less for the OCDR mechanism than for the 'Permanent Connection' mechanism. This can be partially explained by the fact that OCDR works with a higher value of: $\mu$. If a connection is present, the OCDR mechanism can transfer data at a higher rate, and is therefore less sensitive to higher loads. For low loads, the connection availability decreases slightly, so that $E T$ does not decrease, as is the case for the 'Permanent Connection' mechanism.

In Figure 9, it can be seen that the average reserved bandwidth decreases for very low load. For $\lambda>10$ it is almost constant. This indicates that the OCDR mechanism is stable with these parameters: the connection is available during bursts and released between bursts, regardless of the traffic intensity during the burst.

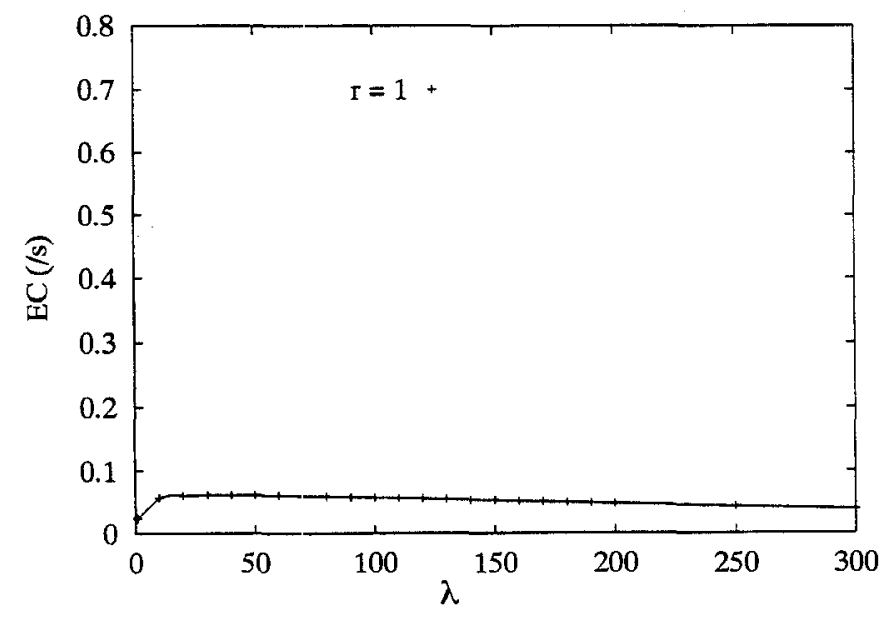

Figure 10: Average number of connection setups per second

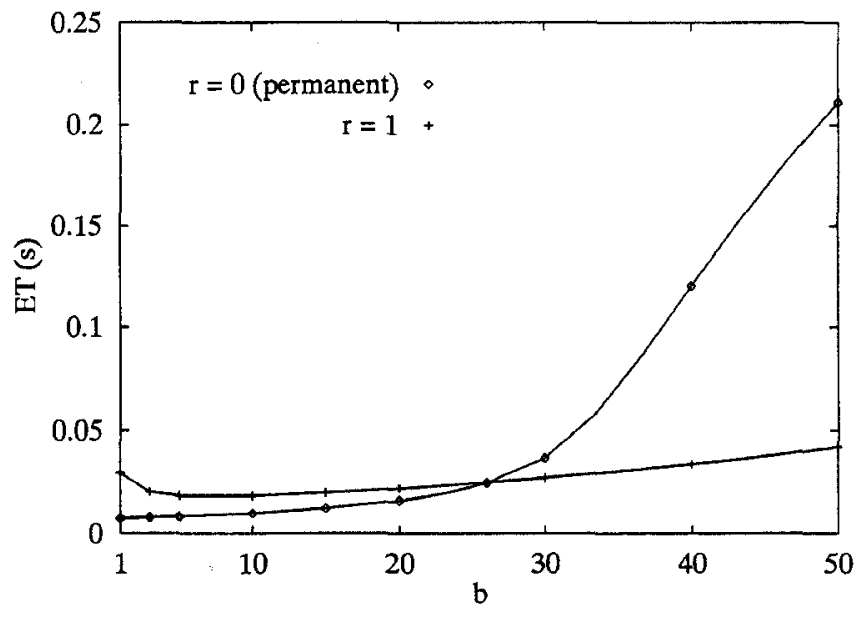

Figure 11: Average node delay under varying burstiness

Figure 10 confirms this indication. The average number of connection setups per second does not vary much under varying load. For the given parameters, a connection is established approximately every 20 seconds.

\section{Behaviour under varying burstiness}

Next, we want to examine the behaviour of the OCDR under varying burstiness. Here, the burstiness $b$ will be defined as:

$$
b=\frac{\text { arrival rate in burst }}{\text { mean arrival rate }}=\frac{\lambda}{(\lambda \beta) /(\alpha+\beta)}=\frac{\alpha+\beta}{\beta}
$$

The burstiness $b$ will be varied by varying $\alpha, \beta$, and $\lambda$, while keeping the mean time between the start of two consecutive bursts $(1 / \alpha+1 / \beta)$ and the mean arrival rate $((\lambda \beta) /(\alpha+\beta))$ constant. This means that the burst length is varying, but the average number of arrivals per burst is constant. Again, graphs for the average node delay ET (Figure 11 ), the average reserved bandwidth $E B W$ (Figure 12), and the average number of connection setups per second $E C$ (Figure 13) are given.

From Figure 11, it can be observed that the OCDR mechanism is also not very sensitive to varying burstiness w.r.t.

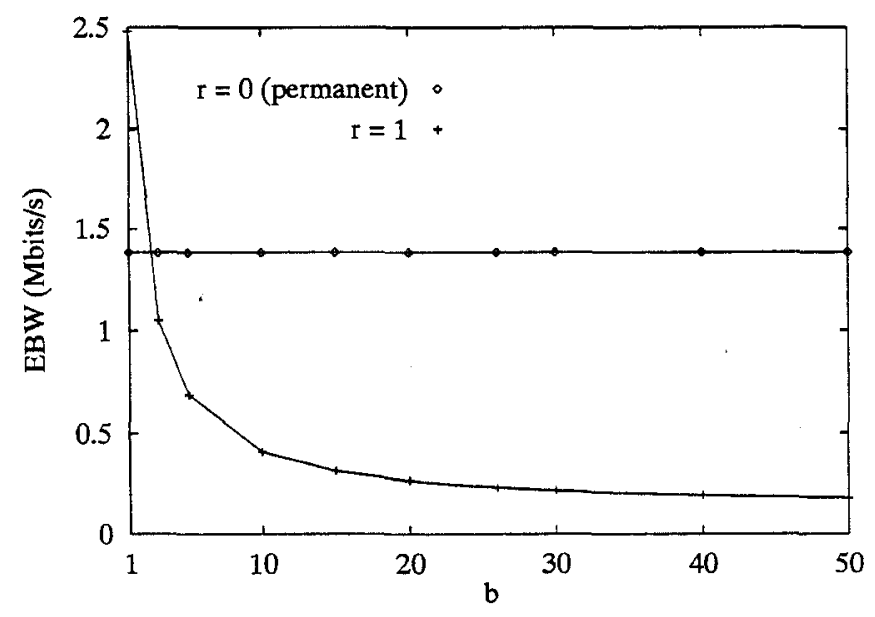

Figure 12: Average reserved bandwidth under varying burstiness 


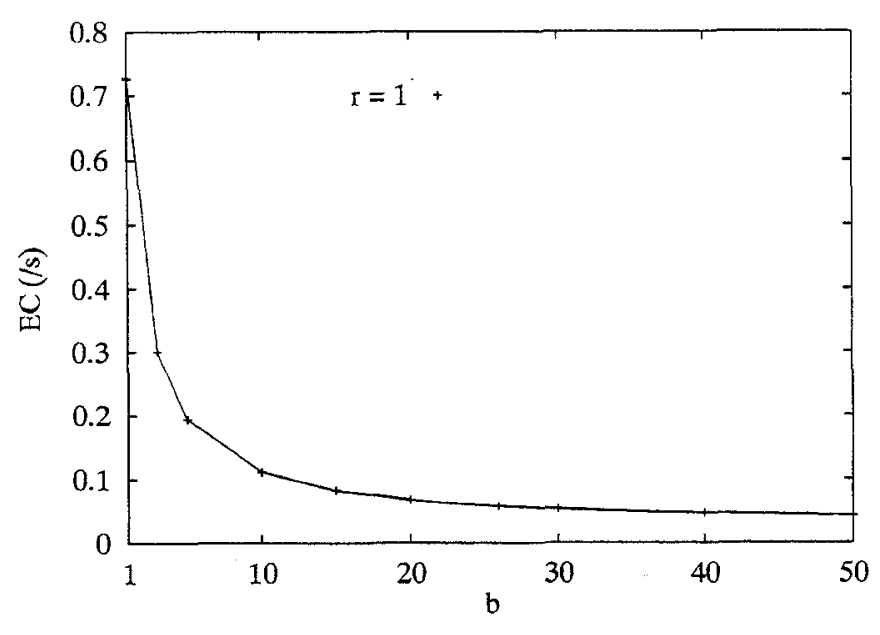

Figure 13: Average number of connection setups per second under varying burstiness

average node delay. Again, this can be explained by the higher transfer rate during connection availability. For a very low burstiness, $E T$ grows. If $b \rightarrow 1$, the system approximates the behaviour under Poisson traffic (Figure 6). OCDR is then no longer advantageous.

It can be concluded that burstiness is an essential factor for the well operation of OCDR. In Figure 12, it can be seen that the burstiness should be sufficiently high to obtain a significant bandwidth reduction. This can be explained by the fact that optimally, a connection is available during a burst, and released during the interburst period. As a result, the reserved bandwidth will be proportional to the fraction of time, the user is generating bursts.

Figure 13 indicates that a high burstiness is also needed to avoid a high number of connection setups per second. This is due to the fact that for the given parameters the traffic intensity becomes so low for low burstiness, that the connection is often released during a burst.

The major conclusions of this evaluation are that the OCDR mechanism is advantageous if the offered traffic has a bursty nature. Furthermore, the traffic intensity during a burst should not be too high, since all packets arriving during the connection setup period have to be buffered. This makes the mechanism most suitable for situations where $\mathrm{CL}$ protocol entities in the end system of individual users have to be connected to intermediate $\mathrm{CL}$ protocol entities. In situations where intermediate protocol entities have to be interconnected, or where LANs have to be connected to intermediate protocol entities, the burstiness will probably be low, and the load high, because of the large number of communicating users.

The advantages that can be offered by the OCDR mechanism are the following:

- significant bandwidth reductions can be obtained, and

- the obtained average node delay is not strongly related to the burstiness and intensity of the offered traffic.

\section{Conclusions}

We have identified the problem of providing a connectionless service using an underlying connection oriented service. Four mechanisms which can provide a solution for this problem have been described. A promising one, the OCDR mechanism, has been analysed using Markov models. Two models have been constructed: one for Poisson traffic load, and one for a more realistic bursty traffic load. For the first Markov model, a closed form solution has been found. The second one has been solved numerically.

The analyses reveal that significant bandwidth reductions can be obtained provided that the offered traffic has a bursty nature. Furthermore, the OCDR mechanism has the advantageous property that the obtained average node delay is not strongly related to the intensity and burstiness of the offered traffic.

Future work includes the analysis of the Markov model for bursty traffic load with matrix geometric solution methods. A closed form solution of the model would give more insight in the behaviour of OCDR, and allow for a better optimization of the system parameters.

\section{Acknowledgements}

The authors would like to thank Boudewijn R. Haverkort, Hans Daemen, and Henk Jan Olde Loohuis, who have contributed to the work presented in this paper.

\section{References}

[1] Byrne, W.R., Clapp, G., Kafka, H.J., Luderer, G.W.R., Nelson, B.L., "Evolution of Metropolitan Area Networks to Broadband ISDN", IEEE Communications Magazine, Vol. 29, No. 1, January 1991, pp. 69 - 82.

[2] Hemrick, C.F., Lang, L.J., "Introduction to Switched Multi-megabit Data Service (SMDS), an early broadband service", Proceedings XIII International Switching Symposium (ISS '90), Stockholm, May 1990, pp. 1 - 8.

[3] CCITT, Recommendations drafted by working party XVIII/8 (General B-ISDN aspects) to be approved in 1990", CCITT SG XVIII, Geneva, May 1990.

[4] Heijenk, G.J., Niemegeers, I.G., "Variable Bandwidth Connections for a Connectionless Service on ATM - Performance Modelling and Evaluation", Proceedings IFIP Workshop on Broadband Communications, Elseviers Science Publishers, Estoril, Portugal, January 1992.

[5] Jain, R., Routhier, S.A., "Packet Trains - Measurements and a New Model for Computer Network Traffic", IEEE Journal on Selected Areas in Communications, Vol. 4, No. 6, September 1986, pp. 986 - 995.

[6] Gusella, R., "Characterizing the Variability of Arrival Processes with Indexes of Dispersion", IEEE Journal on Selected Areas in Communications, Vol. 9, No. 2, February 1991, pp. $203-211$. 